Andrews University

Digital Commons @ Andrews University

Honors Theses

Undergraduate Research

4-1-2019

\title{
Acoustic Reflex and Extended High-Frequency Testing in Relation to Tinnitus
}

Deborah Kim

Andrews University, deborahk@andrews.edu

Follow this and additional works at: https://digitalcommons.andrews.edu/honors

Part of the Speech Pathology and Audiology Commons

\section{Recommended Citation}

Kim, Deborah, "Acoustic Reflex and Extended High-Frequency Testing in Relation to Tinnitus" (2019).

Honors Theses. 210.

https://dx.doi.org/10.32597/honors/210

https://digitalcommons.andrews.edu/honors/210

This Honors Thesis is brought to you for free and open access by the Undergraduate Research at Digital Commons @ Andrews University. It has been accepted for inclusion in Honors Theses by an authorized administrator of Digital Commons @ Andrews University. For more information, please contact repository@andrews.edu. 


\title{
J. N. Andrews Honors Program \\ Andrews University
}

HONS 497

Honors Thesis

Acoustic Reflex and Extended High-Frequency Testing in Relation to Tinnitus

\author{
Deborah S. Kim
}

1 April 2019

Advisor: Darah Regal Au.D., CCC-A

Primary Advisor Signature: Dawh Rezal

Department: Speech-Language Pathology and Audiology 


\begin{abstract}
Tinnitus, a growing concern among adults, is the perception of sound without an external acoustic source. Tinnitus can sound like ringing, buzzing, and clicking and is typically caused by noise-induced hearing loss. Noise-induced hearing loss is caused by prolonged exposure to high levels of noise, which damages the cochlea, and can be temporary or permanent. The purpose of this study is to determine if there are any common factors or characteristics in Acoustic Reflex, standard pure tone and/or Extended High-Frequency testing results between two groups: people who report and do not report tinnitus. This study explores whether Acoustic Reflex testing and Extended HighFrequency audiometric testing results will provide quantitative evidence of ear pathology associated with tinnitus.
\end{abstract}




\section{INTRODUCTION}

The purpose of this study is to test Acoustic Reflex thresholds and perform Extended High-Frequency audiometry on individuals who report tinnitus based on completion of a validated questionnaire called the "Tinnitus Handicap Inventory" (see Methodology). Tinnitus, which is the perception of sound when no actual noise is present, is a growing complaint among collegiate students. Tinnitus can manifest itself as anything from ringing, buzzing, and clicking, and is typically caused by noise-induced hearing loss. Noise-induced hearing loss is caused by prolonged exposure to high levels of noises, which damages the hair cells of the cochlea, and can be temporary or permanent. According to the American Academy of Family Physicians, noise can cause permanent hearing loss at chronic exposures equal to an average SPL (Sound Pressure Level) of $85 \mathrm{~dB}$ or higher for an 8-hour period. The amount of time that is considered safe for listening decreases as the decibel level is increased by $3 \mathrm{~dB}(\mathrm{~A}) ; 85 \mathrm{~dB}(\mathrm{~A})$ will permit you to listen safely for 8 hours, while $88 \mathrm{~dB}(\mathrm{~A})$ results in 4 hours of safe listening (Noise-Induced, 2012). The World Health Organization reported that a staggering 1.1 billion teenagers and young adults are at risk for hearing loss due to misuse of personal audio devices and exposure to venues such as nightclubs, bars, sporting events, and music concerts (Sminkey, 2015). Noise-induced hearing loss is devastating to physical and mental health, education, and employment, so it is essential to be proactive in its prevention. It is a "common opinion among researchers within the field of audiology" that due to more sources of noise exposure in today's society, the prevalence of tinnitus will increase (Widen \& Erlandsson, 2004). 
The physiological causes of tinnitus have been unknown until more recently, as studies are gradually showing what is the cause of the disorder by examining the ear at the cochlear level and neural level. According to the Journal of Clinical Neurology, tinnitus does not represent a disease itself but is actually a symptom of a variety of underlying diseases (Han et al, 2009). The goal of this study is to further examine auditory tinnitus by evaluating the Acoustic Reflex threshold, Extended High-Frequency thresholds and the Tinnitus Handicap Inventory of a group of patients.

\section{ACOUSTIC REFLEX DEFINED}

The Acoustic Reflex is a protective feature of the inner ear that protects the ear from extremely loud sounds. The stapedius muscle, the smallest muscle of the body, contracts in response to loud sounds and is responsible for preserving our hearing. Without this reflex, hearing ability would deteriorate quickly due to the prevalence of loud and sudden sounds in society. The Acoustic Reflex is a bilateral reflex - the stapedius muscles of both the left and right ear are stimulated regardless of what ear you put a loud sound in. Thus, the Acoustic Reflex consists of two pathways - the ipsilateral pathway and the contralateral pathway. The pathway begins at the outer ear, travels through the middle ear, through the cochlea, and then is conducted along the eighth auditory nerve to the brainstem. The impulse is received by the cochlear nucleus and superior olivary complex in the brain stem. From there, the seventh facial nerve innervates the right and left stapedius muscle, evoking the Acoustic Reflex in the ear. One would not anticipate the Acoustic Reflex at the normal conversational level, which is around 40-60 dB HL. Usually if a stimulus is between 70 to 100 decibels, the Acoustic 
Reflex is activated. If the reflex occurs outside of this range, there is a potential problem in the ear, typically in the cochlea or the acoustic nerve (CN VIII). For example, a response at a low sensation (lower than $70 \mathrm{~dB}$ ) indicates a cochlear problem and a response at a high sensation level (higher than $100 \mathrm{~dB}$ ) indicates a retrocochlear problem. Tinnitus can occur due to a cochlear or retrocochlear pathology. The Acoustic Reflex testing may give an indication regarding the possible location or origin of the tinnitus.

\section{EXTENDED HIGH-FREQUENCY THRESHOLDS DEFINED}

Pure tone audiometry evaluates the hearing threshold of individuals at different frequencies. The hearing threshold is defined as the softest sound level in decibels a person can hear $50 \%$ of the time. For a normal adult ear, a threshold should be between -10 to $25 \mathrm{~dB}$ for all frequencies. Typically, hearing tests are only done between 250 to $8000 \mathrm{~Hz}$. Thus, Extended High-Frequency audiometry includes frequencies from 9000 to $20000 \mathrm{~Hz}$. The 9000 to $20000 \mathrm{~Hz}$ range are affected earlier on in life before the standard $250-8000 \mathrm{~Hz}$ range. Previous undergraduate research performed by Trine and Rosu at Andrews University have concluded that those with tinnitus also exhibit increased thresholds in the higher frequencies. Thus, it may be concluded that testing EHF can be used for early detection for individuals with tinnitus. Furthermore, studies from the American Journal of Otolaryngology show that individuals of 15-30 years have increased thresholds in the Extended High-Frequencies as a result of using personal listening devices (Kumar et al, 2017).

According to a study published by American Journal of Audiology, previous research has indicated elevated or absent Acoustic Reflex thresholds in subjects with 
complaints of tinnitus (Fernandes et al, 2013). In conjunction with this finding, previous undergraduate students under Dr. Darah Regal have found that the mean threshold of Extended High-Frequency thresholds are higher for people who have tinnitus while absent otoacoustic emissions did not have any correlation with tinnitus. (An otoacoustic emission is a phenomenon in which the cochlea, in response to being stimulated with a sound, emits a sound back out of the ear that can be measurable and predictable). Thus the purpose of this study is to demonstrate whether both Extended High-Frequency thresholds and Acoustic Reflex thresholds are elevated in those with tinnitus.

Expected benefits from this research study will be to alert participants to potential risk factors of hearing loss, as well as detect early hearing loss in the high frequencies as the 9000 to $20000 \mathrm{~Hz}$ range are affected earlier on in life than the standard 250 to 8000 Hz range. This study should help clarify possible tests that correlate with tinnitus, cochlear versus retro-cochlear, using Acoustic Reflex and Extended High-Frequency testing. It is expected that the severity of the tinnitus will directly correlate to hearing loss in EHF and elevated AR. Furthermore, college-age adults are complaining more and more of ringing in their ears possibly due to noise-induced hearing loss. This exposure to loud sounds typically causes cochlear damage, which is what results in tinnitus. Thus, if an audiologist can detect the beginning signs of cochlear damage from EHF and AR threshold testing, patient counseling regarding appropriate protection of the cochlea may result in preservation of healthy hearing (Audiologic Guidelines, 2000).

Thus, this study will involve conducting Acoustic Reflex and Extended Highfrequency testing on two groups of adults: first, those who complain of tinnitus, and second, a control group. The goal is for Acoustic Reflex to be elevated/absent and 
Extended High-Frequency threshold to be higher in those who complain of tinnitus, thus clarifying possible audiological tests that could be used to alert patients of possible auditory system damage which may be related to auditory tinnitus. Since both Acoustic Reflex and Extended High-Frequency audiometry is an important tool for the differential diagnosis of tinnitus in individuals, this research will help provide more specificity on additional tests that may indicate an auditory tinnitus and will give audiologists more information regarding hearing-loss prevention. A combined study of AR and EHF could assist in educating individuals about noise-induced hearing loss and the early effects that noise exposure can have upon the cochlea.

PRIMARY RESEARCH QUESTIONS

- Can both Acoustic Reflex testing (AR) and Extended High-Frequency (EHF) audiometric testing results provide quantitative evidence of possible ear pathology associated with tinnitus in young adults?

\section{SECONDARY RESEARCH QUESTIONS}

- Is there a correlation between absent or elevated AR thresholds and higher EHF thresholds and will this correlation help us to differentiate between those with low tinnitus scores versus high tinnitus scores on the tinnitus questionnaire?

- Will both AR and EHF audiometry together, provide numeric data to counsel patients with tinnitus concerning auditory/cochlear function? 


\section{METHODOLOGY}

This study was a quantitative non-experimental comparative and predictive design and was open to all adults 18 or older who reported having tinnitus. There were 24 individuals (ages 20-83) with self-reported symptoms of tinnitus and a control group of 20 individuals. A peer-reviewed and validated questionnaire called the "Tinnitus Handicap Inventory" was used as a way of qualifying people for the research study. The severity scale at the end of the survey ranked the grades of tinnitus from slight to catastrophic. A complete hearing evaluation was conducted to detect thresholds outside the range of normal hearing. Hearing within normal limits was defined as hearing thresholds of -10 to $-25 \mathrm{~dB}$ HL for adults. Extended High-Frequency (EHF) threshold testing was carried out to detect high frequency losses that may be present, as well as tympanometry and Acoustic Reflex (AR) testing. Data was collected from July 2017 thru January 2019. Numeric values from AR and EHF were compared to the Tinnitus Questionnaire percentage results to determine any correlational components.

Instrumentation: Andrews University's Department of Speech-Language Pathology and Audiology had the necessary and appropriate equipment for this research study, including a sound isolated chamber, annually calibrated audiometer including Extended High-Frequency, and immittance bridge for Acoustic Reflex. A valid and peerreviewed Tinnitus Questionnaire was utilized, with an attached form for contact information and consent statement for research study (see end of Methodology section for copy of the questionnaire). This Questionnaire was brief, easy to administer and interpret, broad in scope, and included a "Tinnitus Handicap Inventory Severity Scale" with 5

grades corresponding to increasing degrees of tinnitus. A file number was assigned on the 
first page and then entered on each subsequent page of the case history form in order to preserve anonymity.

Data collection procedures: Each participant was scheduled for a pure tone air threshold testing at $250-18000 \mathrm{~Hz}$ in a sound isolated chamber at Andrews University's Department of Speech-Language Pathology and Audiology. An informed consent form was completed as well. Only the participant number/code appeared on all testing materials (tinnitus severity data, pure tone, and AR results). The testing was conducted and/or supervised by Darah Regal, AuD, CCC-A. The hearing test consisted of wearing earphones and raising their hand in response to a pure tone ranging in frequency from $250-18000 \mathrm{~Hz}$. This type of testing did not present risk to the patient and followed specified guidelines using the bracketing technique for obtaining threshold testing. Hearing threshold testing was a subjective test because the patient's response is the criteria for determining if a tone was heard. Additionally, Tympanometry and Acoustic Reflex Testing were done to each participant. For this objective test, a probe was placed in the outer part of the ear and ear canal volume, pressure, and tympanic membrane mobility will be measured. Next Acoustic Reflex threshold was determined. Acoustic Reflex testing determined the lowest decibel level at which the patient's stapedius muscle contracts. The same probe was placed in the patient's outer ear canal and stimulated the ear and measured the ear's Acoustic Reflex threshold at $1000 \mathrm{~Hz}$. Then another probe was placed in the patient's other ear canal and measured the Acoustic Reflex threshold at $1000 \mathrm{~Hz}$ of the other ear. This test was an objective test and requires no response from the patient. The patient sat quietly while a pressure transducer and microphone stimulates and measures each ear. This portion took approximately 10 minutes. Both pure-tone 
audiometry and Acoustic Reflex testing are standard procedures that are done regularly in the SPLAD clinic as a part of a complete hearing evaluation.

Data analysis: Descriptive statistics (independent t-test) were used to describe the variables and the participants. Numeric values from AR and EHF were compared to the Tinnitus Questionnaire percentage results to determine any correlational components. Levene's Test for Equality of Variances was used to assume equal variances. Numeric values from AR and EHF were compared to the Tinnitus Questionnaire results to determine any correlation between absent or elevated AR thresholds and higher EHF thresholds. The goal was to determine whether this correlation help audiologists differentiate between those with low versus high tinnitus scores. 
TINNITUS HANDICAP INVENTORY

Patient Name:

Date:

INSTRUCTIONS: The purpose of this questionnaire is to identify difficulties that you may be experiencing because of your tinnitus. Please answer every question. Please do not skip any questions.

\begin{tabular}{|c|c|c|c|}
\hline 1. Because of your tinnitus, is it difficult for you to concentrate? & Yes & Sometimes & No \\
\hline 2. Does the loudness of your tinnitus make it difficult for you to hear people? & Yes & Sometimes & No \\
\hline 3. Does your tinnitus make you angry? & Yes & Sometimes & No \\
\hline 4. Does your tinnitus make you feel confused? & Yes & Sometimes & No \\
\hline 5. Because of your tinnitus, do you feel desperate? & Yes & Sometimes & No \\
\hline 6. Do you complain a great deal about your tinnitus? & Yes & Sometimes & No \\
\hline 7. Because of your tinnitus, do you have trouble falling to sleep at night? & Yes & Sometimes & No \\
\hline 8. Do you feel as though you cannot escape your tinnitus? & Yes & Sometimes & No \\
\hline $\begin{array}{l}\text { 9. Does your tinnitus interfere with your ability to enjoy your social activities } \\
\text { (such as going out to dinner, to the movies)? }\end{array}$ & Yes & Sometimes & No \\
\hline 10. Because of your tinnitus, do you feel frustrated? & Yes & Sometimes & No \\
\hline 11. Because of your tinnitus, do you feel that you have a terrible disease? & Yes & Sometimes & No \\
\hline 12. Does your tinnitus make it difficult for you to enjoy life? & Yes & Sometimes & No \\
\hline 13. Does your tinnitus interfere with your job or household responsibilities? & Yes & Sometimes & No \\
\hline 14. Because of your tinnitus, do you find that you are often irritable? & Yes & Sometimes & No \\
\hline 15. Because of your tinnitus, is it difficult for you to read? & Yes & Sometimes & No \\
\hline 16. Does your tinnitus make you upset? & Yes & Sometimes & No \\
\hline $\begin{array}{l}\text { 17. Do you feel that your tinnitus problem has placed stress on your relationships } \\
\text { with members of your family and friends? }\end{array}$ & Yes & Sometimes & No \\
\hline $\begin{array}{l}\text { 18. Do you find it difficult to focus your attention away from your tinnitus and } \\
\text { on other things? }\end{array}$ & Yes & Sometimes & No \\
\hline 19. Do you feel that you have no control over your tinnitus? & Yes & Sometimes & No \\
\hline 20. Because of your tinnitus, do you often feel tired? & Yes & Sometimes & No \\
\hline 21. Because of your tinnitus, do you feel depressed? & Yes & Sometimes & No \\
\hline 22. Does your tinnitus make you feel anxious? & Yes & Sometimes & No \\
\hline 23. Do you feel that you can no longer cope with your tinnitus? & Yes & Sometimes & No \\
\hline 24. Does your tinnitus get worse when you are under stress? & Yes & Sometimes & No \\
\hline \multirow[t]{4}{*}{ 25. Does your tinnitus make you feel insecure? } & Yes & Sometimes & No \\
\hline & \multicolumn{3}{|c|}{ FOR CLINICIAN USE ONLY } \\
\hline & & & \\
\hline & $\times 4$ & $x 2$ & xo \\
\hline Total Score & & + & \\
\hline
\end{tabular}

\section{TINNITUS HANDICAP INVENTORY SEVERITY SCALE}

\begin{tabular}{|c|c|l|}
\hline GRADE & SCORE & DESCRIPTION \\
\hline 1 & $0-16$ & $\begin{array}{l}\text { Slight: Only heard in quiet environment, very easily masked. No interference with sleep or daily } \\
\text { activities. }\end{array}$ \\
\hline 2 & $18-36$ & $\begin{array}{l}\text { Mild: Easily masked by environmental sounds and easily forgotten with activities. May } \\
\text { occasionally interfere with sleep but not daily activities. }\end{array}$ \\
\hline 3 & $38-56$ & $\begin{array}{l}\text { Moderate: May be noticed, even in the presence of background or environmental noise, } \\
\text { although daily activities may still be performed. }\end{array}$ \\
\hline 4 & $58-76$ & $\begin{array}{l}\text { Severe: Almost always heard, rarely, if ever, masked. Leads to disturbed sleep pattern and can } \\
\text { interfere with ability to carry out normal daily activities. Quiet activities affected adversely. }\end{array}$ \\
\hline 5 & $78-100$ & Catastrophic: Always heard, disturbed sleep patterns, difficulty with any activity. \\
\hline
\end{tabular}




\section{RESULTS}

First examining Extended High-Frequency threshold results, the mean was found by combining all of the decibel thresholds in the tinnitus group and all of the decibel thresholds in the control group. Figures 1 and 2 display the mean pure tone thresholds values for the control and tinnitus groups. The group with tinnitus had higher pure tone thresholds for almost every frequency than the control group. Pure tone results were analyzed and revealed a significant difference between the control and tinnitus groups. The sample means, t- and p- values are displayed in Figure 4, which show that subjects in the control group had significantly lower mean pure tone thresholds in specific frequencies in both the right and the left ear than the tinnitus group. There is statistical evidence that the associated population means are significantly different at the 0.05 level. As a side note, there were two individuals in the control group and six individuals with tinnitus who did not respond to even the highest decibels during the pure tone audiometry evaluation (NR). Thus, the limits of the audiometer were used in determining the following means. The limits of the audiometer are as follows:

$$
\begin{gathered}
9000 \mathrm{~Hz}-100 \mathrm{~dB} \\
10000 \mathrm{~Hz}-105 \mathrm{~dB} \\
11200 \mathrm{~Hz}-105 \mathrm{~dB} \\
12500 \mathrm{~Hz}-100 \mathrm{~dB} \\
14000 \mathrm{~Hz}-90 \mathrm{~dB} \\
16000 \mathrm{~Hz}-75 \mathrm{~dB} \\
18000 \mathrm{~Hz}-35 \mathrm{~dB}
\end{gathered}
$$


Figure 1: Threshold Means for Standard Pure Tone and EHF Tests in Right Ear

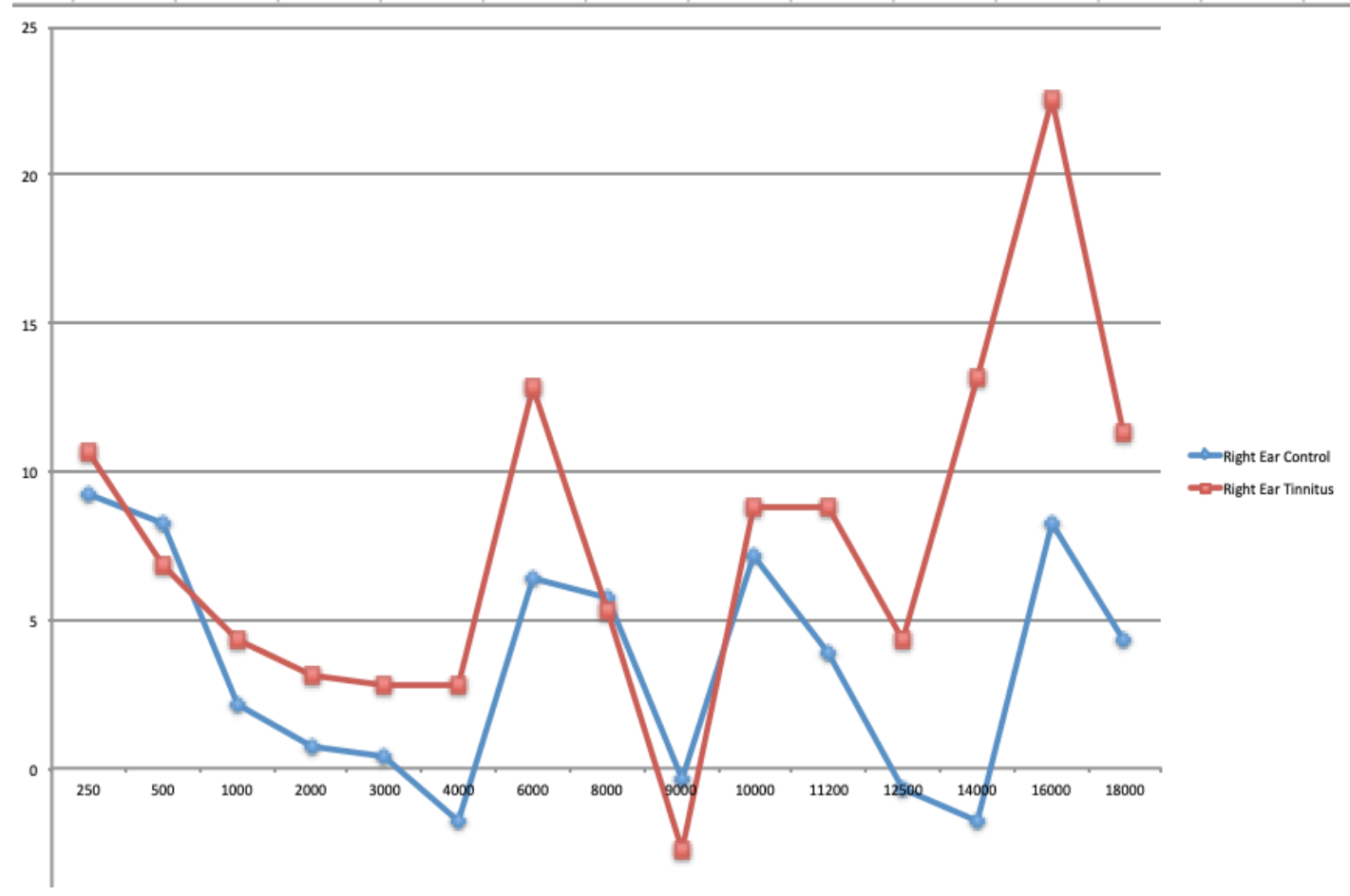

Figure 2: Threshold Means for Standard Pure Tone and EHF Tests in Left Ear

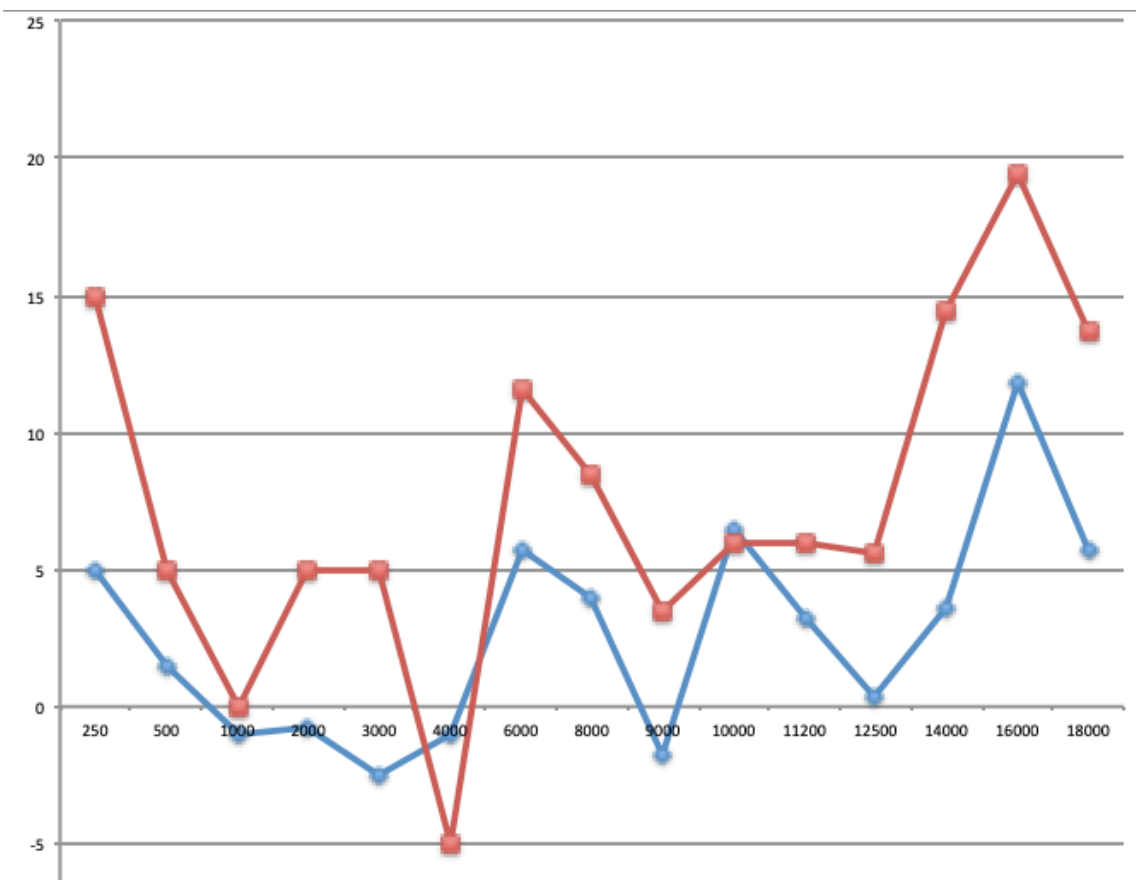

$\rightarrow$ Left Ear Control $\rightarrow$ - Left Ear Tinnitus 
Figure 3: Threshold Means with Frequencies Divided into Bands

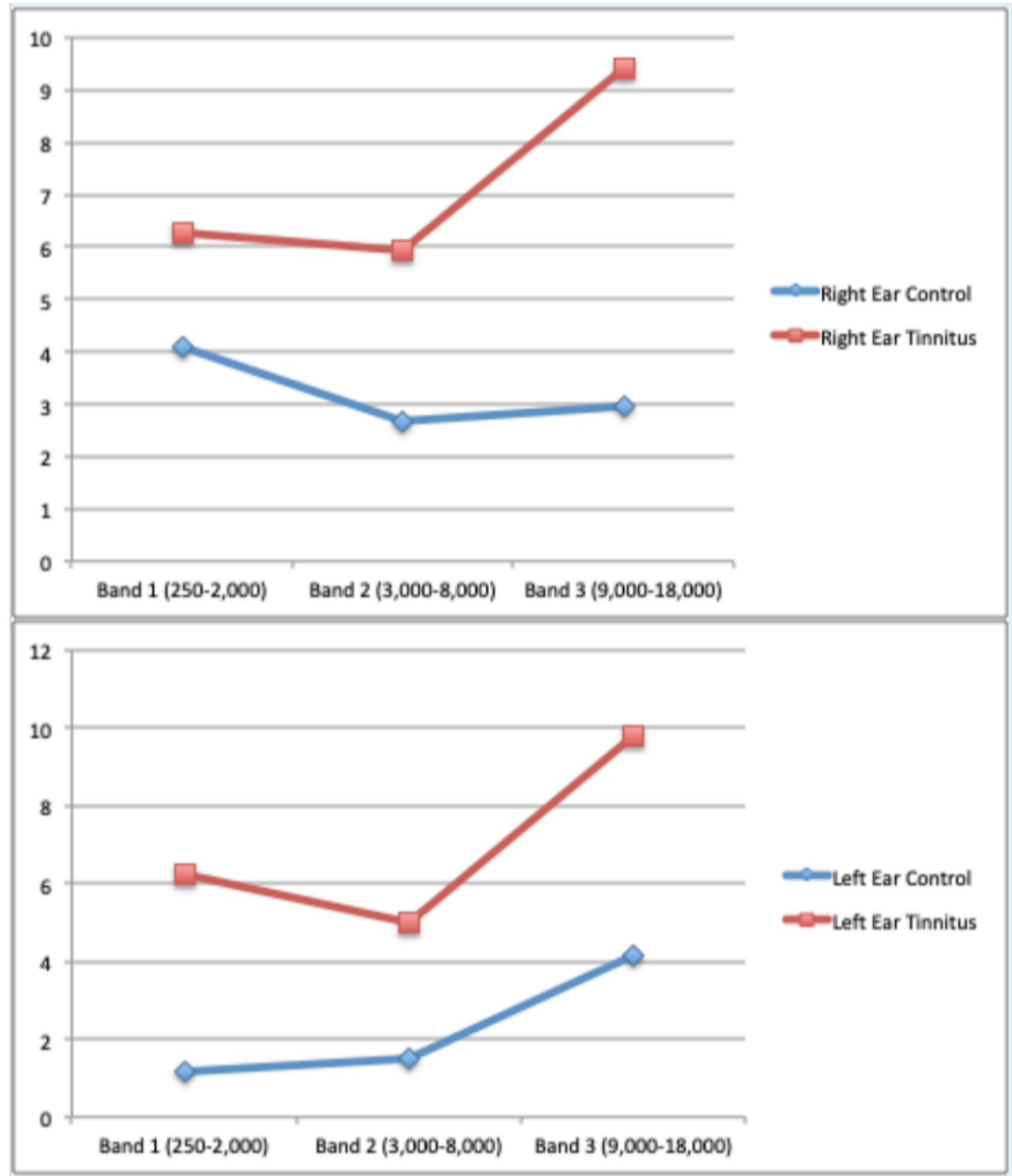

The individual frequencies from Figure 1 and Figure 2 were also divided into bands in order to determine whether certain ranges of frequencies were affected more than other areas. According to Figure 3, each band is significantly different between the control and the tinnitus, especially in the Extended High-Frequency (9000-18000) range. 
Figure 4: T-test for Equality of Means for Different Bands

\begin{tabular}{llllll}
\hline Frequency & Mean & Std. Dev. & t & df & Sig. (2-tailed) \\
\hline RBAND1 C & 4.3472 & 4.07660 & -3.126 & 40 & .003 \\
RBAND1 T & 8.5417 & 4.46403 & & & \\
RBAND2 C & 6.9444 & 11.22319 & -1.654 & 40 & .106 \\
RBAND2 T & 14.8542 & 17.77561 & & & \\
RBAND3 C & 10.9524 & 20.00300 & -2.094 & 40 & .043 \\
RBAND3 T & 25.8036 & 24.56933 & & & \\
RBAND4 C & 10.8333 & 12.74755 & -2.015 & 40 & .051 \\
RBAND4 T & 21.8750 & 20.42018 & & & \\
RBAND5 C & 26.65441 & 26.65441 & -2.232 & 40 & .031 \\
RBAND5 T & 28.35384 & 28.35384 & & & \\
\hline
\end{tabular}

\begin{tabular}{llllll}
\hline Frequency & Mean & Std. Dev. & t & df & Sig. (2-tailed) \\
\hline LBAND1 C & 2.0833 & 4.94455 & -2.239 & 40 & .031 \\
LBAND1 T & 7.1458 & 8.56526 & & & \\
LBAND2 C & 6.0417 & 11.54634 & -1.912 & 40 & .063 \\
LBAND2 T & 15.6354 & 18.75949 & & & \\
LBAND3 C & 8.6806 & 15.31545 & -2.154 & 40 & .037 \\
LBAND3 T & 23.2887 & 25.50357 & & & \\
LBAND4 C & 10.2778 & 13.11400 & -2.008 & 40 & .051 \\
LBAND4 T & 21.2500 & 20.17586 & & & \\
LBAND5 C & 23.0556 & 28.60167 & -1.245 & 40 & .220 \\
LBAND5 T & 33.9583 & 27.70101 & & & \\
\hline
\end{tabular}

Key:

- Band 1:250-3000 Hz

- Band 2: 3000-8000 Hz

- Band 3: $9000-18000 \mathrm{~Hz}$

- Band 4: $6000 \mathrm{~Hz}$

- Band 5: $16000 \mathrm{~Hz}$

In previous studies, individuals with tinnitus were found to have a noise notch, or higher threshold, at $6000 \mathrm{~Hz}$ and $16000 \mathrm{~Hz}$, which explains why Band 4 and Band 5 were included.

In addition, no correlation was found between age and the "Tinnitus Handicap Inventory" score (Pearson Correlation $=-.014)$, meaning that older age did not indicate higher severity of tinnitus in this study. 
Next, the Acoustic Reflex threshold results were analyzed. Figure 5 demonstrates that no correlation was found between the absent or elevated Acoustic Reflex thresholds and higher Extended Frequency thresholds. Although it was suspected that those with tinnitus would have abnormal Acoustic Reflex thresholds compared to the control group, there was no correlation in this area either. Even though both sensation level (dB SL) and hearing level (dB HL) were analyzed, there was no correlation found between having tinnitus and higher Acoustic Reflex thresholds. [Note: In the "hearing level" scale (HL), the lowest sound intensity that stimulates normal hearing is "zero decibel hearing level" or dB HL. Audiometers are calibrated in dB HL. On the other hand, the reference for sensation level (SL) is the person's own threshold. For example, an individual may hear a $4000 \mathrm{~Hz}$ tone at $30 \mathrm{~dB} \mathrm{HL}$. If their threshold at $4000 \mathrm{~Hz}$ is $5 \mathrm{~dB} \mathrm{HL}$, then their sensation level would be $25 \mathrm{~dB}$ SL (30 subtracted by 5).]

Figure 5: Acoustic Reflex Results (Control vs. Tinnitus) in Sensation Level (SL)

\section{Acoustic Reflex Results (Control vs. Tinnitus) in Sensation Level}

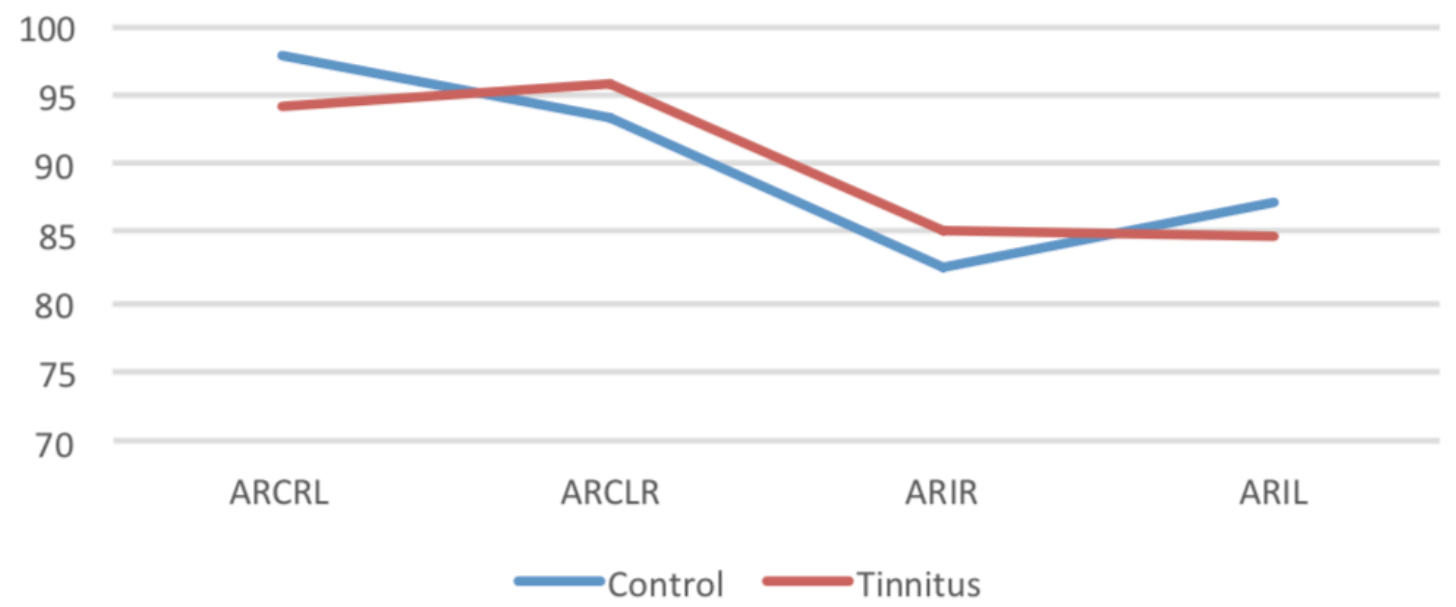




\section{DISCUSSION}

Standard pure tone and Extended High-Frequency threshold results revealed a significant difference between the control and tinnitus groups. The subjects in the control group had significantly lower mean pure tone thresholds in specific frequencies in both the right and the left ear. There is statistical evidence that the associated population means are significantly different in the following areas at the alpha $=0.05$ level:

- In both ears, individuals with tinnitus had higher hearing thresholds for Band 1 $(250-2000 \mathrm{~Hz})$ than the control group $($ Right $\mathrm{p}=.003$, Left $\mathrm{p}=.031)$

- In both ears, individuals with tinnitus had higher hearing thresholds for Band 3 $(9000-18000 \mathrm{~Hz})$ than the control group (Right $\mathrm{p}=.043$, Left $\mathrm{p}=.037)$

- In the right ear, individuals with tinnitus had higher hearing thresholds for Band 5 $(16000 \mathrm{~Hz})$ than the control group (Right $\mathrm{p}=.031)$

Conclusively, Extended High-Frequency audiometric testing results does provide quantitative evidence of ear pathology associated with tinnitus especially in the low frequencies $(250-2000 \mathrm{~Hz})$ and the higher frequencies $(9000-18000 \mathrm{~Hz})$. Acoustic Reflex testing does not provide evidence, as there is no correlation between absent or elevated AR thresholds and higher EHF thresholds.

In conjunction with this finding, previous undergraduate students under Darah Regal, Au.D., CCC-A, have found that absent otoacoustic emissions did not have any correlation with tinnitus while the mean threshold of Extended High-Frequency thresholds are higher for people who have tinnitus. This study once again confirmed that auditory tinnitus can be clarified by higher Extended High-Frequency thresholds but found that tinnitus has no correlation to absent or elevated Acoustic Reflex thresholds. 
This research study is beneficial as it helps to alert participants to potential risk factors to hearing loss as well as detect early hearing loss in the Extended HighFrequencies (the 9000 to $20000 \mathrm{~Hz}$ range is affected earlier on in life than the standard $250-8000 \mathrm{~Hz}$ range). This study was able to clarify possible tests that correlate with tinnitus, revealing that although Extended High-Frequency testing is helpful, Acoustic Reflex testing is not. Thus, if an audiologist detects the beginning signs of cochlear damage through EHF threshold testing, patient counseling regarding appropriate protection of the cochlea may result in preservation of healthy hearing. Since it has been shown that Extended High-Frequency audiometry is an important tool for the differential diagnosis of tinnitus in individuals, this research provides more specificity on which tests may indicate an auditory tinnitus and which tests will not.

The limitations of this study are that there was an unbalanced age representation in the sample. Further studies are recommended to include more individuals in the sixty to eighty year old ranges in both the control group and tinnitus group, as there were very few individuals in this age range represented in this study. This will ensure that the correlation coefficient between age and the Tinnitus Handicap Inventory score will be more accurate. In addition, when testing Acoustic Reflex, this study only evaluated the Acoustic Reflex threshold at $1000 \mathrm{~Hz}$ due to time constraints. In the future, Acoustic Reflex threshold results at $500 \mathrm{~Hz}, 2000 \mathrm{~Hz}$, and $4000 \mathrm{~Hz}$ should also be included in order to confirm whether or not there is no correlation between higher Acoustic Reflex thresholds in those with tinnitus than those in the control group.

A potential future study is to conduct a pre-test and post-test (dependent t-test) on college students who have recently been exposed to loud sounds the night or two nights 
before such as in a concert or other loud environment. The test would determine whether there is a bigger threshold shifts immediately after the concert and a return to normal a week or a month after the concert. This future study could ask the participants to follow a few pointers to restore their hearing (i.e., reduced caffeine consumption, increased water intake, staying out of noise, abstaining from earphones) and evaluate how effective these treatment options are. 


\section{BIBLIOGRAPHY}

Han, B.; Lee, H.; Kim, T.; Lim, J.; Shin, K. Tinnitus: Characteristics, Causes, Mechanisms, and Treatments. Journal of Clinical Neurology. 2009, 11-19. https://www.ncbi.nlm.nih.gov/pmc/articles/PMC2686891/

This article examines the several theories that have been proposed to explain the mechanisms underlying tinnitus and has concluded that tinnitus represents a symptom, rather than a single disease. The researchers have found that tinnitus occurs in both the auditory pathway and the peripheral auditory system, and treatments include pharmacotherapy, cognitive and behavioral therapy, sound therapy, music therapy, massage and stretching. This article is pertinent to my research because I will be furthering an aspect of Han's study by specifying further the physiological causes of tinnitus.

Fernandes C.; Momensohn-Santos, T.; Carvalho J. Tinnitus and normal hearing: a study on contralateral acoustic reflex. National Center for Biotechnology Information, U.S. National Library of Medicine. 2013, 291-6. https://www.ncbi.nlm.nih.gov/pubmed/23824442

This aim of this study was to compare adult subjects with hearing within normal limits, with and without tinnitus and evaluate how the contralateral acoustic reflex threshold is affected. When the stapedius muscle of the 7 th nerve contracts in response to a loud sound, this is a bilateral reflex - thus, there is both an ipsilateral 
and contralateral pathway. When a loud sound occurs, both the left and right ear reflexes are activated regardless of which side the loud sound occurred on. The study showed that elevated or absent acoustic reflex thresholds were found only in subjects with complaints of tinnitus. These results are pertinent to my research because I will be examining whether both the acoustic reflex thresholds and the extended high-frequency thresholds are elevated in individuals with tinnitus.

Bauman, N. What are Safe Sound Levels? Center for Hearing Loss Help. Retrieved from http://hearinglosshelp.com/blog/what-are-safe-sound-levels/.

This study provides a systemized time/loudness scale, which indicates safe sound levels all the way to dangerous sound levels. For each 3-decibel increase in sound level, the time that one can listen is reduced by half. Unprotected exposure to continuous noise above 115 decibels of any duration is not permitted. These sound levels give explanations as to why collegiate-aged students are obtaining noise-induced hearing loss today. This is important for my study because noiseinduced hearing loss usually occurs with tinnitus.

Widen, S.; Erlandsson, S. Self-reported tinnitus and noise sensitivity among adolescents in Sweden. Noise Health. 2004, 7(25), 29-40. Retrieved from https://www.ncbi.nlm.nih.gov/pubmed/15703147. 
This study focuses on using hearing protection and self-reported hearing-related symptoms primarily in a group of high-school students, aged 13 to 19 students. Widen and Erlandsson's results prove that the prevalence of tinnitus will increase as a consequence of environmental factors such as exposure to loud sounds especially during activities such as pop concerts and gyms. One of the goals of my study is to further the prevention of hearing impairments in the young population by promoting the use of hearing protection. Age-related differences in the prevalence of tinnitus and noise sensitivity were significant.

Kumar, P., Upadhyay, P., et al.. Extended high frequency audiometry in users of personal listening devices. American Journal of Otolaryngologist. 2017, 38(2). Retrieved from https://www.ncbi.nlm.nih.gov/pubmed/27979368 .

Kumar et al.'s study is pertinent to my research as elevated hearing thresholds were observed at extended-high frequencies $(9 \mathrm{kHz}, 10 \mathrm{kHz}, 11 \mathrm{kHz}, 13 \mathrm{kHz}, 14$ $\mathrm{kHz}, 15 \mathrm{kHz}$, and $16 \mathrm{kHz}$ ) for individuals who frequently used personal listening devices. Especially users who had used listening devices for more than five years at a high volume showed significant increase in hearing thresholds. This research concluded that extended high frequencies can be used for early detection of noiseinduced hearing loss. These results bolster my research study as I examine whether both acoustic reflex thresholds and extended high-frequency thresholds are elevated in people with tinnitus. 
Audiologic Guidelines for the Diagnosis \& Management of Tinnitus Patients. American Academy of Audiology. 2000. Retrieved from http://www.audiology.org/publications-resources/document-library/audiologicguidelines-diagnosis-management-tinnitus-patients.

This article is important to my study because its discussion on the diagnosis and management of patients with tinnitus connects to one of the goals of my research study - which is to spread awareness and strategies for hearing prevention. This research study is not just a biomedical orientation about the audiogram or the physiological characteristics of tinnitus, but it is also a patient-centered approach in which we are concerned for the individual's long-term hearing health. This article by the American Academy of Audiology lists tinnitus patient management procedures such as counseling, cognitive behavioral therapy, habituation, and stress management concerning tinnitus.

Sminkey, L. 1.1 billion people at risk for hearing loss. World Health Organization. 2015. Retrieved from http://www.who.int/mediacentre/news/releases/2015/ear-care/en/.

Sminkey's article on the World Health Organization website depicts the grave reality of the exponential rise in young people affected by noise-induced hearing loss. This is pertinent to my research as noise-induced hearing loss leads to tinnitus. One overarching purpose of my study is to educate and counsel individuals who are at risk for noise-induced hearing loss and those who already 
experience tinnitus. $50 \%$ of teenagers and young adults aged $12-35$ years old tested by WHO are exposed to unsafe levels of sound from personal audio devices and $40 \%$ are exposed to environments such as entertainment venues that cause noise-induced hearing loss. This has led to worsened physical and mental health and difficulties in education and employment.

Noise Induced Hearing Loss. American Hearing Research Foundation. 2012. Retrieved from http://american-hearing.org/disorders/noise-induced-hearing-loss/\#causes.

This article is important to my research as tinnitus is largely caused by noiseinduced hearing loss. My sample size consists of collegiate-aged students. Due to increase in use of personal listening devices, more and more young adults are experiencing noise-induced hearing loss. This article defines the hearing disorder outlines the causes of noise-induced hearing loss, and gives information on how noise induced hearing loss can be diagnosed and treated. 